\title{
Programmed Multi-Level Ventilation: A Strategy for Ventilating Non-Homogenous Lungs
}

\author{
Filip Depta $\mathbb{D}^{1,2}$ \\ Pavol Torok ${ }^{1,2}$ \\ Veldon Reeves ${ }^{3}$ \\ Michael Gentile ${ }^{3}$ \\ 'Department of Critical Care, East Slovak \\ Institute of Cardiovascular Diseases, \\ Košice, Slovakia; ${ }^{2}$ Pavol Jozef Šafárik \\ University, Košice, Slovakia; ${ }^{3}$ Department \\ of Administration and Medical Affairs, \\ IPM Chirana Inc, Durham, NC, USA
}

\begin{abstract}
Mechanical ventilation (MV) has been an integral method used in ICU care for decades. MV is typically viewed as a life-supporting intervention. However, it can also contribute to lung injury through stress and strain, as evidenced by ventilator-induced lung injury (VILI), even in previously healthy lungs. The negative impact may be worsened when significant lung non-homogeneity is present, eg. ALI and ARDS. Protective lung strategies to minimize VILI are to use low tidal volumes (Vt 4-6 mL/kg/PBW), plateau pressures $\left(\mathrm{P}_{\text {plat }}\right)$ $<30 \mathrm{cmH}_{2} \mathrm{O}$ and relatively high positive end-expiratory pressures (PEEP). Yet, use of constantly high PEEP levels is well recognized to result in hemodynamic compromise of the right ventricle, increased stress and strain through high mechanical energy impact on the lung and overdistension of relatively healthy lung tissue. Taking these issues into consideration, a different approach to mechanical ventilation was developed specifically for patients with non-homogeneity. This review focuses on a feature called programmed multi-level ventilation (PMLV). It is not a ventilation mode per se, but rather a form of extension that adjusts and modifies any ventilation mode (eg PCV,PSV, VCV, SIMV, etc.). PMLV is based on measured time constants (Tau) of the whole respiratory system, including artificial airways, breathing circuits, humidification devices and mechanical ventilator. Using a physiology-based approach presents a method to ventilate non-homogenous lungs through cyclic changes of different PEEP levels; recruitment takes place in lung areas with long time constants but protects relatively healthy lung areas from overdistension thus minimizing excessive mechanical power to the lung tissue.
\end{abstract}

Keywords: time constant, positive end expiratory pressure, acute respiratory distress syndrome, programmed multi-level ventilation

\section{Introduction}

Mechanical ventilation (MV) is the life support technique routinely applied for a diverse spectrum of indications, from scheduled surgical procedures to acute organ failure. ${ }^{1}$ It is used when there is impending or existing respiratory failure, impairment in oxygenation, ventilation or a combination of both. Searching for an optimal ventilation strategy for different lung pathologies is one of the most intensely researched fields in critical care medicine. Effort has been put into investigating non-homogeneity of the lung that plays a central role in pathophysiology of acute respiratory distress syndrome (ARDS). ARDS is an inflammatory process in the lungs that induces non-hydrostatic protein-rich pulmonary edema. The immediate consequences are profound hypoxemia, decreased lung compliance, and increased intrapulmonary shunt and increased dead space. $^{2}$ Although research in the field of ARDS has progressed immensely for the past decades, mortality has not changed substantially and remains relatively high depending
Correspondence: Filip Depta

Email fdepta@protonmail.com 
on its severity. ${ }^{3}$ Since 2010, the overall rates of in-hospital, ICU, and 28/30-d, and 60-day mortality were 45, 38, 30, and $32 \%$, respectively. ${ }^{4}$ In survivors, ARDS causes derangement of lung function for 2 years or more after hospital discharge as well as marked reductions in quality of life. ${ }^{5}$

Current recommendations for ARDS ventilated patients include: low tidal volumes $(4-8 \mathrm{~mL} / \mathrm{kg} / \mathrm{PBW})$ and inspiratory pressures $\left(\mathrm{P}_{\text {plat }}<30 \mathrm{cmH}_{2} \mathrm{O}\right)$, prone positioning in refractory hypoxemia, higher vs lower positive end-expiratory pressures (PEEP) and performing lung recruitment maneuvers. ${ }^{6}$ However, recruitment maneuvers are not recommended for routine use due to lack of significant reduction in mortality on day $28 .^{2}$ PEEP remains the cornerstone of ventilation strategies for ARDS. Optimal PEEP is still largely debated and is usually hard to find in routine clinical practice as lung mechanics change during the course of a disease. Apart from extrinsic PEEP that is set deliberately during mechanical ventilation, there also exists intrinsic PEEP (PEEPi) that originates from insufficient expiration time and is generally undesirable.

To address these perplexing issues, programmed multi-level ventilation (PMLV) was developed for use during MV (Aura V, Chirana Medical, Stará Turá, SK). The review describes PMLV used with PCV for mechanically-ventilated patients with non-homogenous lung conditions.

\section{PMLV Physiology}

PMLV incorporates 3 important aspects: time constant (TauE) is measured and optimal frequency suggested accordingly, mathematically-predicted degree of inhomogeneity is incorporated and different PEEP levels are recommended based on calculated compliance.

\section{Time Constant (Tau) Considerations During Mechanical Ventilation and Predicted Optimal Frequency}

Time constant is defined as the time it takes for the lung units to fill or empty. This means that time constant equals the length of time in seconds required for lung units to inflate or deflate to a certain percentage of their total volume. Ventilation of the lung, however, is an exponential process and, by definition, time for lungs to empty after delivering tidal volume takes 3Tau. ${ }^{7}$ In reality, 3 Tau represents $95 \%$ of volume expired and is considered to be a complete process (Figure 1). Tau in respiratory mechanics is a product of compliance of the respiratory system $\left(\mathrm{C}_{\mathrm{st}}\right)$ and airway resistance $\left(\mathrm{R}_{\mathrm{aw}}\right)$. Inspiration and expiration both operate with time constants, but expiratory time constant (TauE) is used as a basis for PMLV, as expiration is presumed to be a passive process even in mechanically-ventilated patients. ${ }^{8}$ Inspiratory time constant is loaded with bias due to triggered breaths or

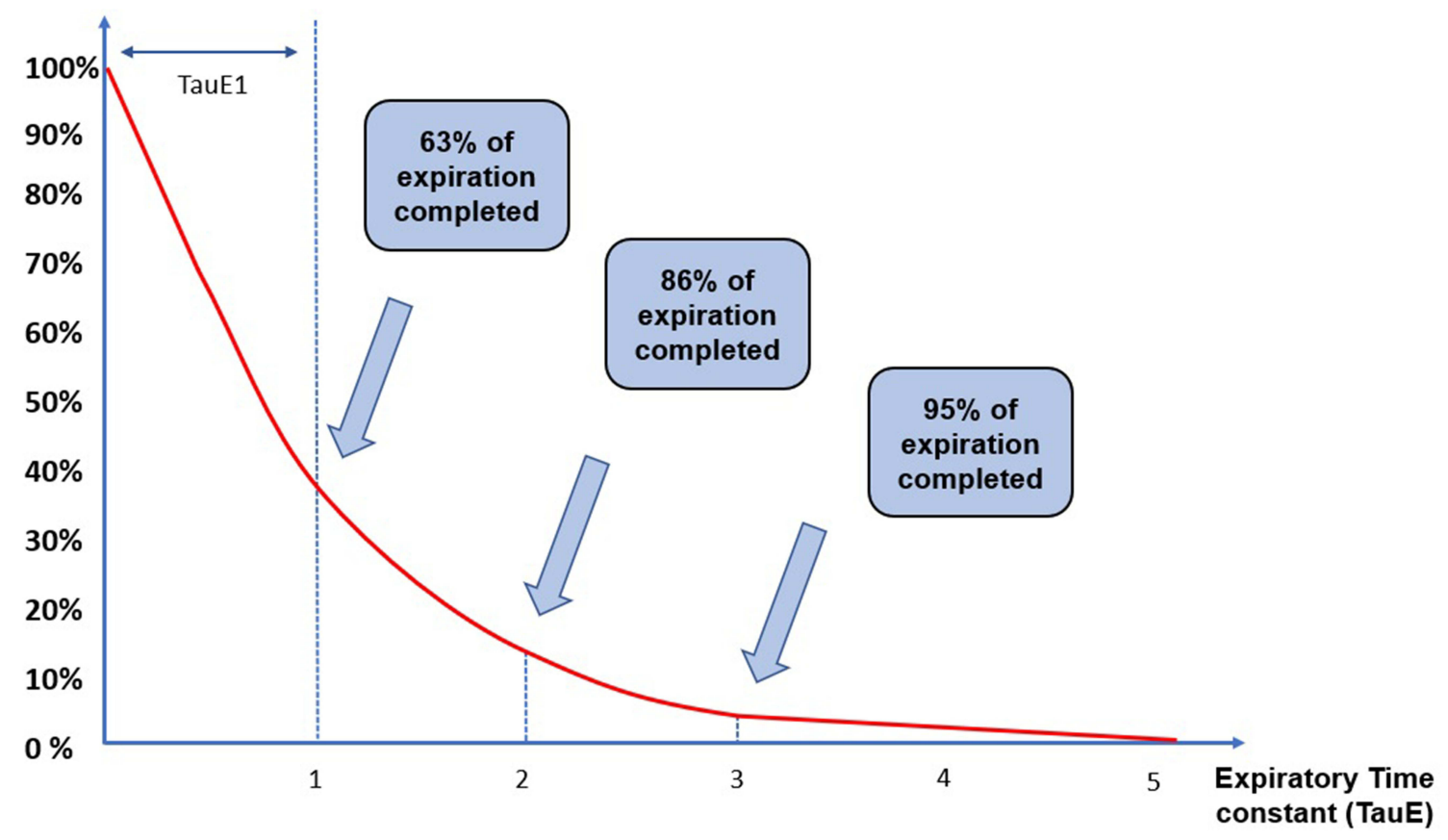

Figure I TauE during expiration with regard to percentage of exhaled volume; $95 \%$ (3TauE) for an exponential process to be fully completed. ${ }^{7}$ 
tachypnea, but mainly because of inspiratory drive. ${ }^{79}$ This theory is even more complicated because time constant is only constant at a particular time. As respiratory mechanics ( $\mathrm{R}$ and $\mathrm{C}$ ) change during the course of a disease, so does the time "constant". Measurements of dynamic expiratory time constant (ie time required for deflation of an end-inspiratory volume by $63 \%$ ) are used for calculation of PMLV.

TauE represents a time constant of the whole respiratory system, including artificial airways, breathing circuits, humidification devices and mechanical ventilators. Time constant in normal healthy lungs typically ranges from $0.6-0.8$ seconds. Values lower than that suggest the restrictive nature of a disease (typically moderate to severe ARDS and fibrotic states), and values above that suggest obstructive conditions (COPD, asthma). ${ }^{10}$

TauE is also used for calculation of respiratory cycle time $\left(\mathrm{T}_{\mathrm{cy}}\right){ }^{8} \mathrm{~T}_{\mathrm{cy}}$ based on measured time constant is calculated as follows:

$$
\mathrm{T}_{\mathrm{cy}}=5.2625 * \mathrm{TauE}+0.1242(1)
$$

Simplified, the above equation can be used to identify optimal frequency $\left(\mathrm{F}_{\mathrm{opt}}\right)$ by multiplying TauE with coefficient 5.4:

$$
\begin{aligned}
& \mathrm{F}_{\text {opt }}=60 / \mathrm{T}_{\text {cy }}(2) \\
& \mathrm{F}_{\text {opt }}=60 /(5.4 \times \text { TauE) (3) }
\end{aligned}
$$

The difference between the calculated and the measured time constant is significant, which makes calculated TauE unusable in clinical practice. ${ }^{8}$ The longer the measured time constant, the lower the "optimal" respiratory rate, and vice versa, to allow full exhalation.

\section{Model-Based Theory of PMLV}

A 5-compartment physical model of diseased lungs is used to demonstrate PMLV and different mechanical properties in terms of resistance (R) and (C). Each of these compartments $(\mathrm{C} 1-\mathrm{C} 5)$ has different $\mathrm{C}$ and $\mathrm{R}$, resulting in different time constant (eg different time needed to fill or empty each compartment). $\mathrm{C} 1$ is the fastest filling compartment with the shortest time constant and $\mathrm{C} 5$ is the compartment with the longest time constant. When PCV (constant inspiratory pressure, one level of PEEP, set respiratory rate, I:E ratio 1:1) is applied to a physical model of nonhomogenous lungs, each compartment is being filled differently (Figure 2).

The same physical model can be used when PMLV is applied using 2 or 3 different levels of PEEP (PEEP, $\operatorname{PEEP}_{h}$ and PEEP $_{h 2}$, first being PEEP level with lowest value and $\mathrm{PEEP}_{\mathrm{h} 2}$ being PEEP level with highest value), which alternate according to a predefined algorithm based on TauE in order to help fill compartments with long time constants.

The difference between PC ventilation (PCV) with one level of PEEP and PCV with an activated PMLV feature (using 2 or 3 levels of different PEEP) in terms of $\mathrm{Vt}$ redistribution from short Tau into long Tau compartments is significant. Using measurements on this mathematical lung model, such Vt redistribution represents about 14\% on average when using 2 PEEP levels (3LV PMLV) and up to $18 \%$ when using 3 different PEEP levels (4LV PMLV). This result may represent a significant shift of a part of $\mathrm{Vt}$ from healthy to diseased compartments in non-homogenous lungs as well (Figures 3 and 4).

\section{Non-Homogeneity of the Lung During PMLV}

Even healthy lungs are non-homogenous to some extent. It has been shown on electric impedance tomography (EIT) images that regional lung aeration differs during mechanical ventilation in healthy patients as well. ${ }^{11}$ This does not represent any clinical problem since oxygen delivery ${ }^{12}$ and $\mathrm{CO}_{2}$ removal meet the physiologic demand. As non-homogeneity increases from ALI to severe ARDS, it becomes a clinical challenge resulting in substantial mortality. ${ }^{4,13}$ Such nonhomogenous lung parenchyma usually presents with bilateral X-ray opacities, CT scan infiltrates with various degree of involvement, and impaired oxygenation. This physiological concept has been described by Gattinoni et al and was termed "baby lung". ${ }^{14}$ Current recommendations for ventilating such ARDS patients focus on protective lung ventilation that has traditionally meant low tidal volumes (Vt of $4-8 \mathrm{~mL} / \mathrm{kg}$ / PBW), PEEP values targeting the oxygenation goal and limiting $\mathrm{P}_{\text {plat }}$ to $30 \mathrm{~cm} \mathrm{H}_{2} \mathrm{O} .{ }^{15}$ However, high PEEP values have their pathophysiologic consequences, eg over-distension of normal lung areas, hemodynamic compromise of the right ventricle, ${ }^{16}$ and increased incidence of barotrauma when not used cautiously. ${ }^{17}$

PMLV, on the other hand, proposes a different concept in order to decrease a degree of non-homogeneity by recruiting long TauE areas of the lung by applying 2 or even 3 levels of PEEP that continuously change. The most pronounced effect of PMLV is recruitment of long TauE areas because subsequent application of higher PEEP provides sufficient pressure and longer inspiratory time for slow regions to fill. When different levels of PEEP change in a step-down approach up to a baseline PEEP it allows enough time to empty long TauE areas. Another aspect is the potentially 

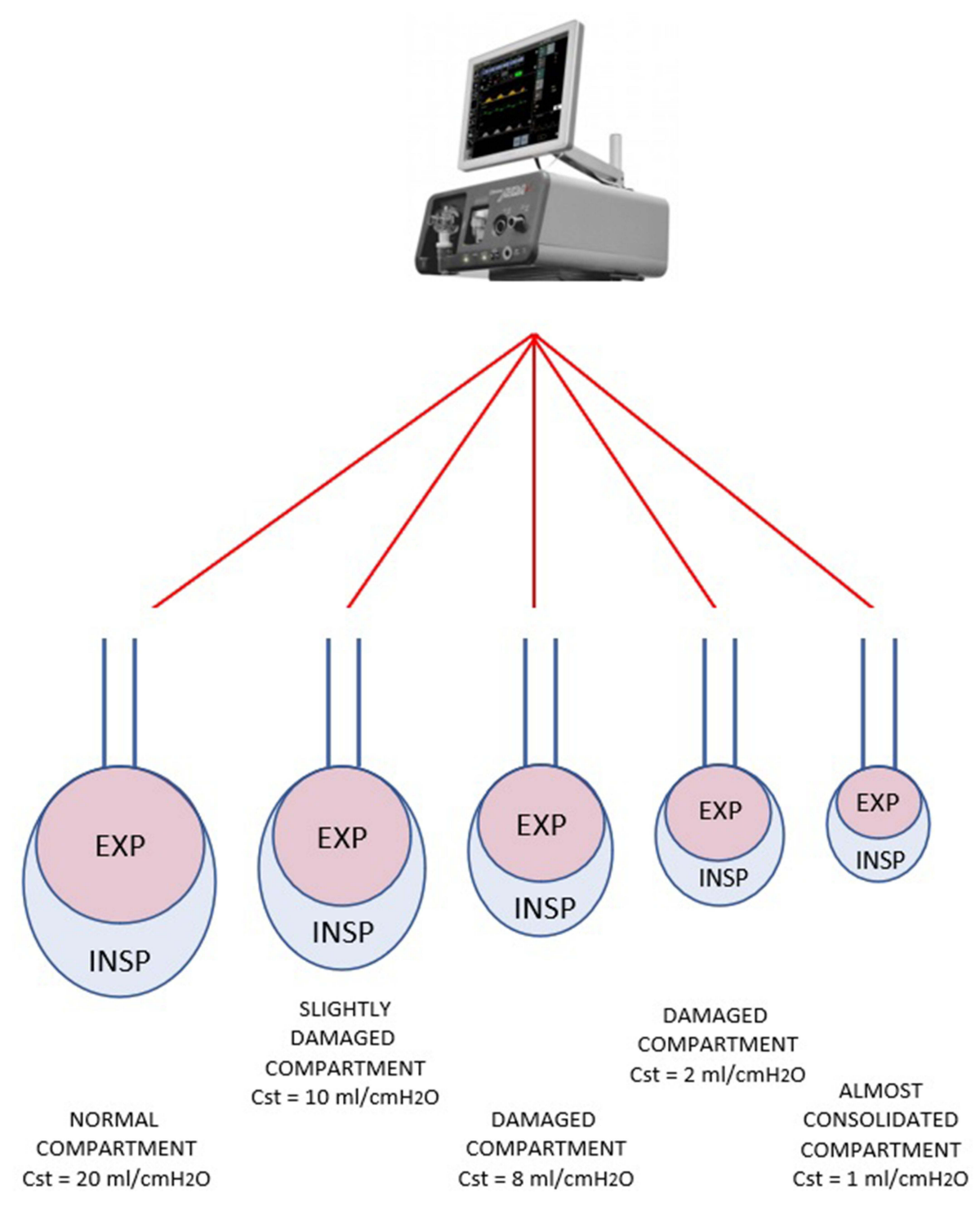

Figure 2 Compartment model showing 5 differently preset compartments with different Tau (different R and C); filling and emptying of each compartment will take different times and hence different optimal frequencies to allow complete filling/emptying $\left(F_{o p t}\right)$.

lower chance of overdistension of healthy areas of the lung parenchyma because the PEEP value is not sustained at high PEEP levels as with conventional strategies.

\section{Lung Recruitment During PMLV}

An open lung approach (OLA) seems well justified at all times during mechanical ventilation of non-homogenous lungs. Recruitment maneuvers (RMs) are transient and sustained increases in transpulmonary pressure are designed to open up collapsed airless alveoli in order to improve gas exchange and compliance, although their role is controversial. ${ }^{18}$ The rationale behind recruitment is justified but it has failed to improve patient mortality. The ART trial showed increased mortality and the PHARLAP trial showed increased cardiovascular adverse events when using recruitment maneuvers. ${ }^{19,20}$ The ART trial used a staircase recruitment maneuver (SRM) with high levels of PEEPs ranging from 25 to $45 \mathrm{cmH}_{2} \mathrm{O}$ with an associated higher chance of VILI and barotrauma. ${ }^{17}$

PMLV, instead, uses a similar approach to staircase recruitment. In this concept, 2 or 3 levels of PEEP are used, together, usually not exceeding 18-20 $\mathrm{cmH}_{2} \mathrm{O}$ through the whole ventilation cycle. The rationale of recruitment with PMLV is based on allowing enough inspiration time for long TauE regions to fill and recruit and the same time to empty when ventilation shifts from highest PEEP through middle PEEP and subsequently to baseline PEEP level (PEEP, PEEP ${ }_{\mathrm{h}}, \mathrm{PEEP}_{\mathrm{h} 2}$ ), respectively. 

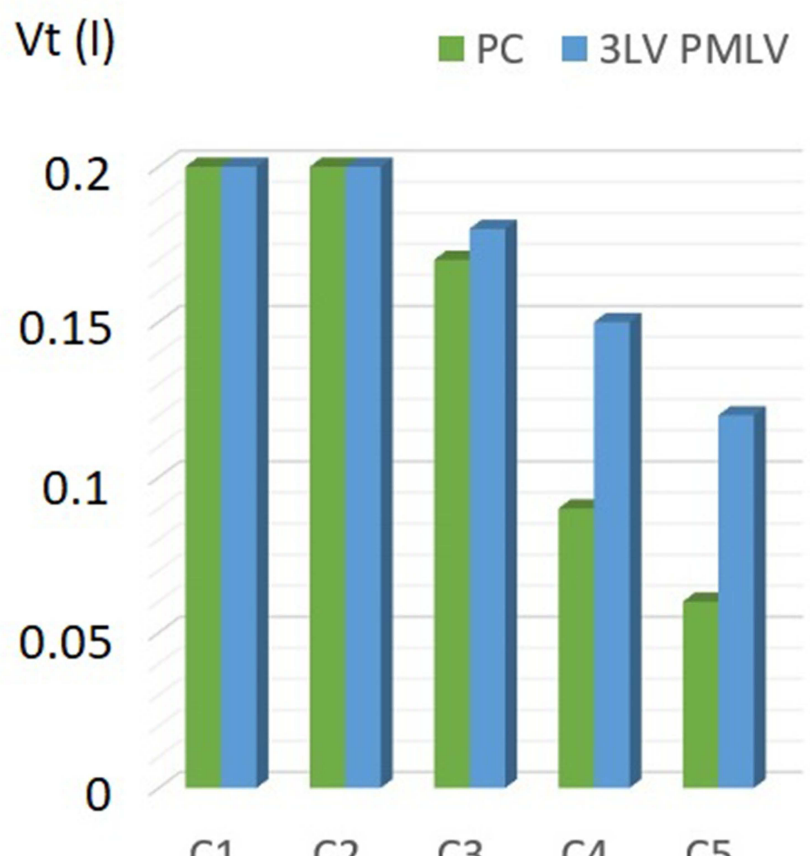

Figure 3 Physical 5-compartment model and mathematically derived values in terms of improved filling of slow compartments with 3LV PMLV. CI-C5 refers to different TauE compartments, $\mathrm{Cl}$ being fastest (shortest TauE) and $\mathrm{C} 5$ slowest (longest TauE). The green color represents $\mathrm{Vt}$ using single PCV with PEEP and blue $\mathrm{Vt}$ with $3 \mathrm{LV}$ PMLV. Marked filling improvement of slow TauE compartments is seen with PMLV.

\section{From Theory to Practice}

It has been described that even the best optimization in terms of frequency and pressure during conventional mechanical lung ventilation strategies cannot optimally distribute gases to differently-diseased compartments. ${ }^{21}$
Hence, a single ventilation mode with fixed ventilation frequency (f), inspiratory time (Ti), I:E ratio, Vt, single PEEP, and inspiratory pressure cannot optimally ventilate inhomogenous lungs. ${ }^{21,22}$ Fixed ventilation parameters will allow optimal ventilation of only some compartments, while others will be either sub-optimally ventilated or over-distended. Using PMLV, these clinical problems may be partly resolved by:

1. Recruitment and ventilation of long TauE areas with high PEEP and low ventilation frequency and also avoiding over-distension of healthy areas (lower PEEP, higher frequency) and thus avoiding potential consequences of sustained high PEEP when conventional ventilation is used (Figure 5).

2. By using measured TauE, intrinsic PEEP (PEEPi) is minimalized by allowing lungs to exhale almost fully.

\section{Algorithm for Setting Up the PMLV}

First, TauE is measured using PCV with a single PEEP for at least 5 minutes until the ventilator averages the TauE and then optimal ventilation frequency is calculated: $\mathrm{F}_{\mathrm{opt}}=$ $60 /(5.4 \times$ TauE $)$. During that time, the ventilator will record the TauE and average this value so that it can be reliably used for PMLV setup. This initial setting is important to remember as it will be used later during re-measuring of TauE - mainly inspiratory pressure (and thus Vt),

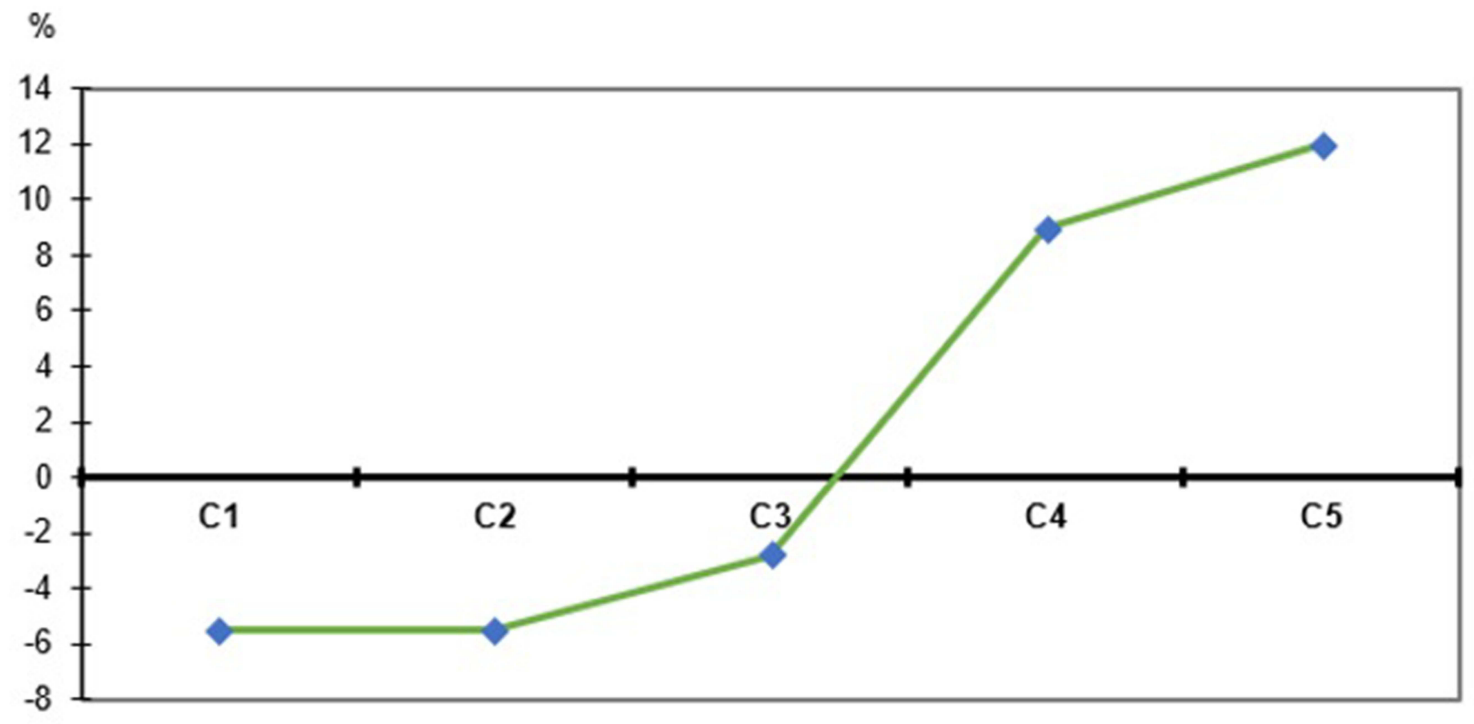

Figure 4 Physical 5-compartment model showing Vt redistribution (\%) from short TauE compartments (CI, C2) into long TauE compartments (C4, C5) using 4LV PMLV. Vt redistribution constitutes $18 \%$, on average. 


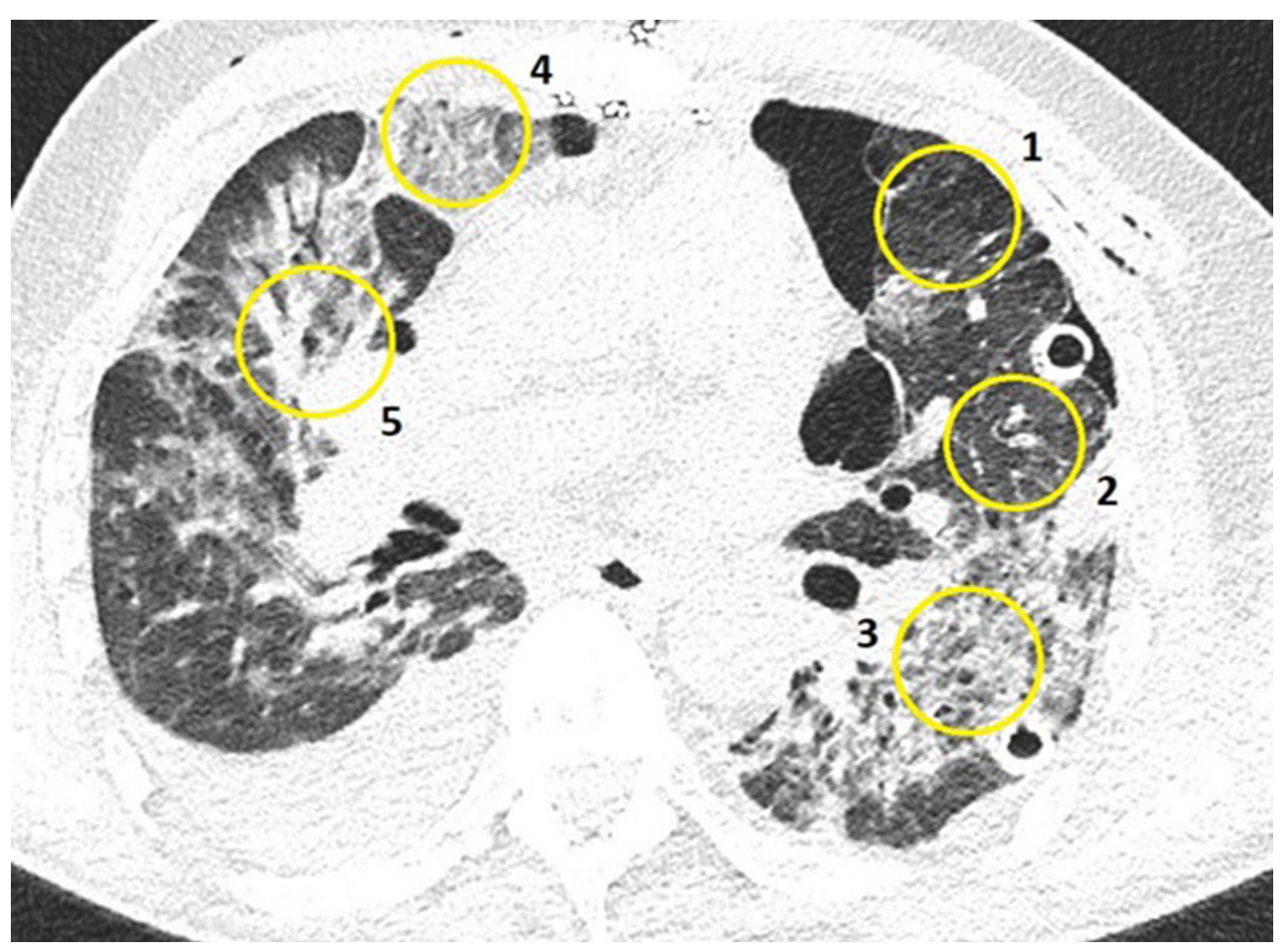

Figure 5 CT scan of ARDS patient showing different areas of non-homogeneity, different theoretical TauE compartments, and hence different optimal ventilation frequencies: I: TauE $0.2 \mathrm{sec}\left(\mathrm{F}_{\mathrm{opt}}=50 \mathrm{bpm}\right) ; 2$ : TauE $0.3 \mathrm{sec}\left(\mathrm{F}_{\mathrm{opt}}=33 \mathrm{bpm}\right) ; 3:$ Tau $0.5 \mathrm{sec}\left(\mathrm{F}_{\mathrm{opt}}=20 \mathrm{bpm}\right) ; 4:$ TauE $0.8 \mathrm{sec}\left(\mathrm{F}_{\mathrm{opt}}=14 \mathrm{bpm}\right) ; 5:$ TauE I.2 sec $\left(\mathrm{F}_{\mathrm{opt}}=8 \mathrm{bpm}\right)$.

frequency, and flow. All these parameters change TauE value if used with different $\mathrm{Vt}$, frequency, and flow. Using different settings when re-measuring TauE would lead to biased TauE and subsequently to different recommended settings of PMLV when lung mechanics change during the course of a disease in the same patient.

Then, a different degree of non-homogeneity is selected by the clinician. The PMLV algorithm will slightly alter the calculated optimal frequency $\left(\mathrm{F}_{\mathrm{opt}}\right)$ to compensate for nonhomogeneity and also suggests frequency of higher PEEP levels $\left(\mathrm{PEEP}_{\mathrm{h}}\right.$ and/or $\left.\mathrm{PEEP}_{\mathrm{h} 2}\right)$. Non-homogeneity models are mathematical models that are supposed to alter the PMLV algorithm based on degree of predicted non-homogeneity (or severity of hypoxemia as dictated by oxygenation index). The first model represents the normal lungs and the last the most severe form of ARDS (Table 1).

A final step involves recommending pressure of particular PEEP levels $\left(\mathrm{PEEP}_{\mathrm{h}}\right.$ and/or $\left.\mathrm{PEEP}_{\mathrm{h} 2}\right)$, as well as the pressure of PCV or PSV applied on top of each PEEP based, again, on mathematical model and calculated patient compliance. Peak inspiratory pressure alarms are adjusted by the clinician.

These processes are followed automatically and clinicians can change any parameter recommended by the ventilator, although changing frequency is not recommended as this would affect the whole PMLV concept based on TauE.

\section{Application of PMLV in Clinical Practice}

A clinical scenario involves a patient with moderate to severe ARDS. Steps to set up the PMLV include: PCV mode, PEEP ( 0.8 to $1 \mathrm{~cm} \mathrm{H}_{2} \mathrm{O}$ for each $10 \mathrm{~kg}$ of PBW ( $>5$ to $<10 \mathrm{cmH}_{2} \mathrm{O}$ ), and peak inspiratory pressure to obtain $\mathrm{Vt}$ of $8-10 \mathrm{~mL} / \mathrm{kg}$ PBW; $\mathrm{FiO}_{2}$ is set to maintain $\mathrm{SpO}_{2}$ of $>88 \%$ and frequency of $18 / \mathrm{min}$ (optimal frequency for initial TauE measurement). TauE is measured most precisely if breaths are not triggered and the patient is passive.

Table I Mathematical Models of Non-Homogeneity to Be Selected by the Clinicians - Calculations of Measured Pulmonary Mechanics (TauE and Cst) are Altered by the Preset Selected to Achieve the Optimal Ventilation for a Particular Patient

\begin{tabular}{|l|c|c|}
\hline \multicolumn{3}{|c|}{ Non-Homogeneity Presets } \\
\hline Preset & Meaning & $\begin{array}{c}\text { Predicted Degree of } \\
\text { Non-Homogeneity }\end{array}$ \\
\hline 1 & Normal lungs & None \\
\hline 2 & Mild ARDS & Mild degree \\
\hline 3 & Moderate ARDS & Moderate degree \\
\hline 4 & Moderate/Severe ARDS & High degree \\
\hline 5 & Severe ARDS & Very high degree \\
\hline
\end{tabular}


$3 \mathrm{LV}$ PMLV or 4LV PMLV is selected and one of 5 presets representing severity of non-homogeneity (eg severity of ALI/ARDS) is selected by the clinician. Based on a selected preset, an algorithm will slightly alter the calculated optimal frequency $\left(\mathrm{F}_{\mathrm{opt}}\right)$ to compensate for non-homogeneity and also suggest frequency of higher PEEP levels $\left(\right.$ PEEP $_{\mathrm{h}}$ and/or PEEP $\left._{\mathrm{h} 2}\right)$.

3LV PMLV is usually selected first (PEEP and $\mathrm{PEEP}_{\mathrm{h}}$ and PCV - same pressure used for both PEEP levels) (Figure 6). After selection of PMLV, measured and derived parameters of lung mechanics are observed. If oxygenation does not improve, 3LV PMLV is turned off and the basic PCV or PSV mode is used again to measure TauE and allowed to stabilize again for at least 3-5 minutes. Then, 4LV PMLV (PEEP, PEEP $_{\mathrm{h}}$, PEEP $_{\mathrm{h} 2}$ and PC/PS on every PEEP level) is activated (Figure 7). During the early course of a disease, TauE is remeasured every 6 to 24 hours, depending on the condition. If lung mechanics improve, $4 \mathrm{LV}$ PMLV is changed back to 3LV PMLV and then to a PCV/PS ventilation when weaning is considered in a step-down approach.

\section{Minute Ventilation and PMLV}

As static compliance changes over time, especially during the early course of ARDS, with constant inspiratory pressure modes $\mathrm{Vt}$ and therefore MV are expected to change. To maintain the desired $\mathrm{MV}$, automatic proportional minute volume (APMV, Chirana Medical, Stará Turá, SK) has been developed. APMV operates as a closed loop that is solely based on modulating inspiratory pressure that alters Vt of the next delivered breaths by $\pm 50 \%$ based on targeted MV. Information to change the pressure for on-coming breaths is gained from the $\mathrm{Vt}$ from previous delivered breaths. Frequency is not modulated when APMV is used. In clinical practice, MV is stable and can be adjusted when PMLV is used in conjunction with APMV.

\section{Current Evidence of PMLV}

The use of PMLV has been recently described as a strategy during lung recruitment after heart surgery. ${ }^{23}$ The study described weaning of cardiac surgical patients after extracorporeal circulation using PCV $(\mathrm{n}=44)$ compared to PCV with 3LV PMLV $(n=44)$. The PMLV group showed improved lung mechanics, oxygenation, carbon dioxide removal and hemodynamic stability compared to the PCV group. ${ }^{23}$ Two studies from Berezhnoí et al included ARDS patients and showed that PMLV improved alveolar ventilation and arterial oxygenation and reduced intrapulmonary shunting, duration of mechanical ventilation and length of stay in the intensive care unit. ${ }^{24}$ Another study from the same authors treated 68 patients with severe non-homogenous lung injury of various etiologies. The control group $(\mathrm{n}=34)$ received conventional volume-controlled and pressure-controlled ventilation modes

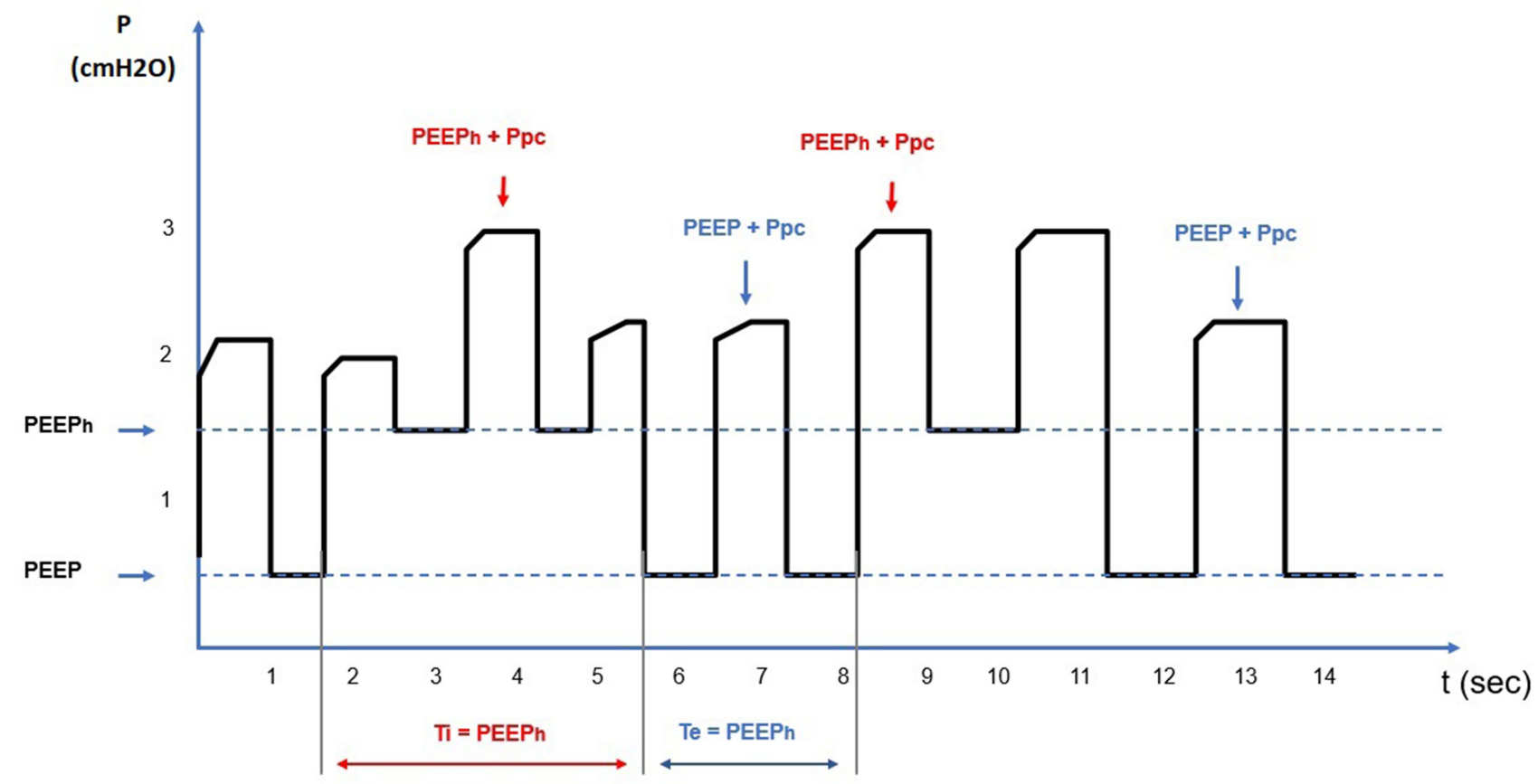

Figure 6 Schematic representation of pressure vs time curve (P/t curve) scheme when 3LV PMLV is activated as an extension mode to PCV, Te (expiratory time), Ti (inspiratory time), $\mathrm{P}_{\mathrm{pc}}$ (pressure of pressure control). 


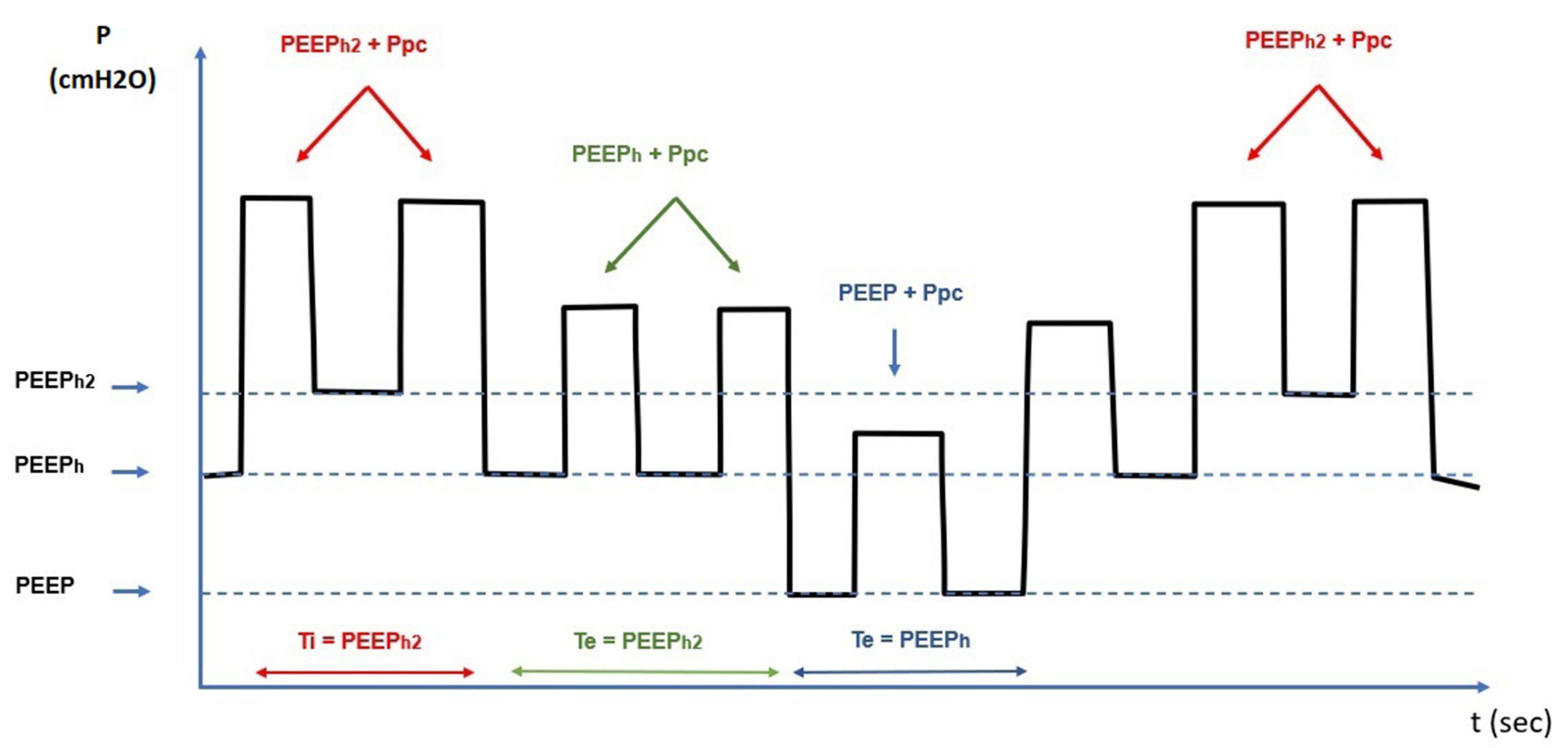

Figure 7 Schematic representation of pressure vs time curve (P/t curve) scheme when 4LV PMLV is activated as an extension mode to PCV, Te (expiratory time), Ti (inspiratory time), $\mathrm{P}_{\mathrm{pc}}$ (pressure of pressure control).

and the PMLV group $(n=34)$ received 3LV PMLV. The PMLV group had improved alveolar ventilation and arterial oxygenation and decreased pulmonary shunting. ${ }^{25}$

\section{Potential Limitations}

Limitation of the PMLV strategy may arise because of a measured variable (TauE) that is adjusted by computed data based on physical and mathematical models, which may be oversimplifications of real non-homogeneity presented in humans.

Additionally, PMLV tries to use the optimal frequency in terms of TauE to a great extent; however, there may be clinical scenarios where lung mechanics will have to be adjusted out of recommended ranges (COPD with extremely long TauE and low recommended respiratory frequency and high Vt in order to maintain MV). In such cases, PMLV is not recommended.

\section{Conclusions and Future Direction}

The knowledge, care and strategies used for ARDS are constantly evolving and being evaluated. The objective of PMLV is to optimize respiratory support while providing protective ventilation to critically ill patients. PMLV may offer advances in terms of recruiting long TauE areas and thus improve oxygenation and $\mathrm{C}_{\mathrm{st}}$, as well as aeration as evidenced by EIT and $\mathrm{CO}_{2}$ elimination, while still utilizing protective ventilation in terms of peak and plateau pressures. PMLV uses physiological principles for the purpose of ventilating non-homogenous lungs, but future studies are needed to assess efficacy in multiple patient populations. Studies focusing on hemodynamic performance, visualizing lung recruitment via EIT and comparing driving pressure $(\Delta \mathrm{P})$ with other ventilation protocols are yet to be conducted. Also, there is a role for both quantitative CT scanning and EIT to evaluate tidal volume distribution and lung homogeneity. These technologies will be incorporated in future clinical trial designs.

\section{Consent for Publication}

All authors read and approved the final manuscript.

\section{Author Contributions}

All authors made a significant contribution to the work reported, whether that is in the conception, study design, execution, acquisition of data, analysis and interpretation, or in all these areas; took part in drafting, revising or critically reviewing the article; gave final approval of the version to be published; have agreed on the journal to which the article has been submitted; and agree to be accountable for all aspects of the work.

\section{Funding}

There is no funding to report. 


\section{Disclosure}

Dr Filip Depta reports personal fees from IPM Chirana Inc. during the conduct of the study. Mr Michael Gentile is affiliated with IPM Chirana Inc. The authors report no other conflicts of interest in this work.

\section{References}

1. Pham T, Brochard LJ, Slutsky AS. Mechanical ventilation: state of the art. Mayo Clin Proc. 2017;92(9):1382-1400. doi:10.1016/j. mayocp.2017.05.004

2. Papazian L, Aubron C, Brochard L, et al. Formal guidelines: management of acute respiratory distress syndrome. Ann Intensive Care. 2019;9(1):69. doi:10.1186/s13613-019-0540-9

3. Parhar KKS, Zjadewicz K, Soo A, et al. Epidemiology, Mechanical power, and 3-year outcomes in acute respiratory distress syndrome patients using standardized screening an observational cohort study. Ann Am Thorac Soc. 2019;16(10):1263-1272. doi:10.1513/ AnnalsATS.201812-910OC

4. Máca J, Jor O, Holub M, et al. Past and present ARDS mortality rates: a systematic review. Respir Care. 2017;62(1):113-122. doi:10.4187/respcare.04716

5. Herridge MS, Cheung AM, Tansey CM, et al. One-year outcomes in survivors of the acute respiratory distress syndrome. $N \mathrm{Engl} \mathrm{J} \mathrm{Med}$. 2003;348:683-693. doi:10.1056/NEJMoa022450

6. Fan E, Brodie D, Slutsky AS. Acute respiratory distress syndrome: advances in diagnosis and treatment. JAMA. 2018;319(7):698-710. doi:10.1001/jama.2017.21907

7. Shevade MS. Time constant: what do we need to know to use it? Indian J Respir Care. 2019;8:4-7. doi:10.4103/ijrc.ijrc_7_18

8. Candik P, Rybár D, Depta F, et al. Relationship between dynamic expiratory time constant tau(edyn) and parameters of breathing cycle in pressure support ventilation mode. Physiol Res. 2018;67 (6):875-879. doi:10.33549/physiolres.933750

9. Guttmann J, Eberhard L, Fabry B, et al. Time constant/volume relationship of passive expiration in mechanically ventilated ARDS patients. Eur Respir J. 1995;8(1):114-120. doi:10.1183/ 09031936.95 .08010114

10. Lourens MS, van den Berg B, Aerts JG, Verbraak AF, Hoogsteden HC, Bogaard JM. Expiratory time constants in mechanically ventilated patients with and without COPD. Intensive Care Med. 2000;11:1612-1618. doi:10.1007/s001340000632

11. Zhao Z, Pulletz S, Frerichs I, Müller-Lisse U, Möller K. The EIT-based global inhomogeneity index is highly correlated with regional lung opening in patients with acute respiratory distress syndrome. BMC Res Notes. 2014;7:82. doi:10.1186/1756-0500-7-82
12. Dunn JOC, Mythen MP. Grocott, Physiology of oxygen transport. BJA Educ. 2016;16:341-348. doi:10.1093/bjaed/mkw012

13. Bice T, Carson SS. Acute Respiratory Distress Syndrome: cost (Early and Long-Term). Semin Respir Crit Care Med. 2019;40(1):137-144. doi:10.1055/s-0039-1685463

14. Gattinoni L, Pesenti A. The concept of "baby lung". Intensive Care Med. 2005;31(6):776-784. doi:10.1007/s00134-005-2627-z

15. Howell MD, Davis AM. Management of ARDS in Adults. JAMA. 2018;319(7):711-712. doi:10.1001/jama.2018.0307

16. Schulman DS, Biondi JW, Zohgbi S, Cecchetti A, Zaret BL, Soufer R. Left ventricular diastolic function during positive end-expiratory pressure. Impact of right ventricular ischemia and ventricular interaction. Am Rev Respir Dis. 1992;145:515-521. doi:10.1164/ajrccm/145.3.515

17. Acosta P, Santisbon E, Varon J. The use of positive end-expiratory pressure in mechanical ventilation. Crit Care Clin. 2007;23 (2):251-261. doi:10.1016/j.ccc.2006.12.012

18. Hess DR. Recruitment Maneuvers and PEEP Titration. Respir Care. 2015;60(11):1688-1704. doi:10.4187/respcare.04409

19. Cavalcanti AB, Suzumura ÉA, Laranjeira LN, Writing Group for the Alveolar Recruitment for Acute Respiratory Distress Syndrome Trial (ART). Effect of Lung Recruitment and Titrated Positive End-Expiratory Pressure (PEEP) vs Low PEEP on Mortality in Patients With Acute Respiratory Distress Syndrome: a Randomized Clinical Trial. JAMA. 2017;318(14):1335-1345. doi:10.1001/ jama.2017.14171

20. Hodgson CL, Cooper DJ, Arabi Y, et al. Maximal Recruitment Open Lung Ventilation in Acute Respiratory Distress Syndrome (PHARLAP). A Phase II, Multicenter Randomized Controlled Clinical Trial. Am J Respir Crit Care Med. 2019;200 (11):1363-1372. doi:10.1164/rccm.201901-0109OC

21. Torok P, Majek M, Kolnik J. Is the Time Constant „Tau (t)” in ALV constant? Theoretical physical model. Anesteziologie a Neodkladná Péce. 2001;6:291-297.

22. Torok P, Majek M. Multilevel Ventilation: theory and Simplified Mathematical Model. General Reanimatology. 2008;4(3):66. doi:10.15360/1813-9779-2008-3-66

23. Candik P, Kolesar A, Nosal M, et al. Use of Programmed Multilevel Ventilation as a Superior Method for Lung Recruitment in Heart Surgery. Int J Crit Care Emerg Med. 2014;5:067.

24. Berezhnoĭ SG, Lukach VN, Glushchenko AV. Respiratory support using multi-level lung ventilation in patients in critical state with systemic inflammatory response syndrome. Anesteziol Reanimatol. 2012;1:55-58.

25. Berezhnoĭ SG, Lukach VN, Tsygankov PV, Malaia NS, Furmanova T. Assessment of oxygen status in critical patients with systemic inflammatory reaction. Anesteziol Reanimatol. $2013 ; 2: 35-40$
Medical Devices: Evidence and Research

\section{Publish your work in this journal}

Medical Devices: Evidence and Research is an international, peerreviewed, open access journal that focuses on the evidence, technology, research, and expert opinion supporting the use and application of medical devices in the diagnosis, monitoring, treatment and management of clinical conditions and physiological processes. The identification of novel devices and optimal use of existing devices which will lead to improved clinical outcomes and more effective patient management and safety is a key feature of the journal. The manuscript management system is completely online and includes a very quick and fair peer-review system. Visit http:// www.dovepress.com/testimonials.php to read real quotes from published authors. 